\title{
Consenso | Protocolo Brasileiro para Infecções Sexualmente Transmissíveis 2020: abordagem às pessoas com vida sexual ativa
}

doi: 10.1590/S1679-4974202100003.esp1

\section{Brazilian Protocol for Sexually Transmitted Infections 2020: approaching sexually active individuals}

\author{
Protocolo Brasileño para Infecciones de Transmisión Sexual 2020: enfoque a las personas \\ con vida sexual activa
}

\author{
Maria Alix Leite Araujo' - (1) orcid.org/0000-0002-4156-5783 \\ Juliana Uesono ${ }^{2}$ - (1) orcid.org/0000-0001-9507-8393 \\ Nádia Maria da Silva Machado² - (1) orcid.org/0000-0001-9334-9305 \\ Valdir Monteiro Pinto ${ }^{3}$ - (1) orcid.org/0000-0002-6880-7607 \\ Eliana Amaral ${ }^{4}$ - (1) orcid.org/0000-0001-9151-3108 \\ 1Universidade de Fortaleza, Programa de Pós-Graduação em Saúde Coletiva, Fortaleza, CE, Brasil \\ ${ }^{2}$ Ministério da Saúde, Secretaria de Vigilância em Saúde, Brasília, DF, Brasil \\ ${ }^{3}$ Prefeitura Municipal de São Paulo, Secretaria Municipal da Saúde, São Paulo, SP, Brasil \\ ${ }^{4}$ Universidade Estadual de Campinas, Faculdade de Ciências Médicas, Campinas, SP, Brasil
}

\section{Resumo}

Este artigo tem como objetivo apresentar conceitos e práticas clínicas recomendados para a abordagem da pessoa com vida sexual ativa. Esses conceitos são parte integrante das recomendações do Protocolo Clínico e Diretrizes Terapêuticas para Atenção Integral às Pessoas com Infecções Sexualmente Transmissíveis (IST) publicado pelo Ministério da Saúde do Brasil em 2020. 0 artigo propõe uma abordagem abrangente da sexualidade para promoção da saúde e apresenta aspectos importantes do processo de comunicação, que deve ocorrer de forma clara, sem preconceitos ou juízos de valor, com foco na saúde sexual e reprodutiva. Destacam-se pontos relevantes acerca do exercício da sexualidade em fases específicas da vida, recomendando avaliação dos riscos e vulnerabilidades, bem como o rastreamento de IST e o uso de preservativos. Dessa maneira, é possível contribuir para que as pessoas possam exercer sua sexualidade de forma plena, responsável e segura.

Palavras-chave: Sexualidade; Infecções Sexualmente Transmissíveis; Prevenção de Doenças; Programas de Triagem Diagnóstica; Protocolos Clínicos; Vigilância.

Endereço para correspondência:

Maria Alix Leite Araujo - Rua São Gabriel, n 300, apto. 1101, Cocó, Fortaleza, CE, Brasil. CEP 60135-450

E-mail:mleite@unifor.br 


\section{Apresentação}

Este artigo tem por objetivo atualizar o capítulo Saúde Sexual: abordagem centrada na pessoa com vida sexual ativa do Protocolo Clínico e Diretrizes Terapêuticas (PCDT) para Atenção Integral às Pessoas com Infecções Sexualmente Transmissíveis (IST) 2020. ${ }^{1}$ Destacam-se os principais pontos da temática, a saber: a comunicação na abordagem da saúde sexual, a sexualidade em fases específicas da vida, a avaliação de riscos e vulnerabilidades, o rastreamento das IST e o uso de preservativos. Foram realizadas adaptações nos itens do capítulo para deixá-lo mais apropriado em relação às questões das outras IST que não o HIV, pois este possui um protocolo clínico específico. ${ }^{2}$

0 PCDT foi publicado pela Secretaria de Vigilância em Saúde, do Ministério da Saúde do Brasil, baseado nas recomendações oficiais e discussões com especialistas e aprovado pela Comissão Nacional de Incorporação de Tecnologias no Sistema Único de Saúde (Conitec) em 2018. ${ }^{3}$ Propõe uma abordagem sensível da sexualidade, visando à melhoria da saúde de pessoas com vida sexual ativa.

\section{Saúde sexual é o estado de bem-estar físico, emocional, mental e social em relação ao exercício da sexualidade, sendo os direitos sexuais e reprodutivos considerados direitos fundamentais.}

\section{Introdução}

Os direitos sexuais e reprodutivos são considerados fundamentais, juntamente com o direito à vida, à alimentação, à saúde, à moradia e à educação para 0 pleno exercício da cidadania. ${ }^{4}$ Reconhece-se o direito de indivíduos e casais de todas as orientações sexuais a ter sua saúde sexual preservada. A saúde sexual é definida como o estado de bem-estar físico, emocional, mental e social em relação a exercício da sexualidade, e não apenas a ausência de doenças sexuais, disfunções ou enfermidades. ${ }^{5}$ É considerada um componente essencial para a promoção do desenvolvimento humano. ${ }^{6}$ Pressupõe o exercício de experiências sexuais seguras e saudáveis, sem coerção, discriminação ou violência. ${ }^{7}$
Por fim, entende-se o sexo como uma das dimensões importantes da sexualidade, que não se limita à genitalidade ou à reprodução. ${ }^{8}$

\section{A comunicação na abordagem da saúde sexual}

0 processo de comunicação exerce um papel preponderante para a melhoria da relação profissional-paciente e, em consequência, para a adesão às recomendações e ao tratamento. ${ }^{9}$ Os profissionais de saúde podem estar despreparados e sentirem constrangimento quando se trata de abordar os pacientes quanto aos aspectos relacionados a doença e sexualidade. ${ }^{10} \mathrm{~A}$ oferta de adequada capacitação poderia minimizar tal constrangimento e contribuir para qualificar o desempenho desses profissionais, visando familiarizá-los com os diferentes conceitos de gênero, orientação e identidade sexual.

Os serviços de saúde devem promover ambientes favoráveis ao diálogo, bem como acolher as diferentes dimensões do exercício da sexualidade de pessoas com vida sexual ativa. Recomenda-se uma abordagem gradual, avançando dos aspectos gerais até os mais específicos. ${ }^{11}$ Uma adequada abordagem da sexualidade deve envolver orientações sobre a prevenção e identificação dos fatores de risco e vulnerabilidades, práticas e comportamentos sexuais que favoreçam 0 contágio por IST.

Para que seja possível estabelecer uma relação de confiança, faz-se necessária uma abordagem clara e transparente, adequada à receptividade e ao contexto de vida das pessoas, que devem ser reconhecidas como sujeitos ativos no processo de cuidado. ${ }^{10}$ Todo 0 atendimento deve favorecer o desenvolvimento da autonomia dos sujeitos para a identificação de soluções às suas demandas. A abordagem deve ocorrer livre de atitudes preconceituosas, rótulos e estigmas, compreendendo a sexualidade como parte da cultura e do contexto histórico, social e de vida de cada indivíduo. ${ }^{4}$

Recomenda-se que o profissional se certifique de que a pessoa está confortável para falar sobre esses temas, avisando que são perguntas realizadas na rotina dos atendimentos, independentemente do sexo, orientação sexual, idade, atividade profissional e estado civil. Deve-se enfatizar o caráter sigiloso e confidencial das informações. ${ }^{12,13}$ Orientações gerais sobre comunicação podem ser encontradas na Figura 1. 
Orientações para a abordagem da sexualidade pelos profissionais de saúde

Estabeleça uma rotina de perguntas a todos os usuários sobre sexualidade (diálogo sobre sexo e práticas sexuais).

Desenvolva seu próprio estilo, atentando para uma relação respeitosa.

Evite julgamentos prévios. Não assuma conceitos prontos (a menos que você pergunte, não há como conhecer a orientação sexual, os comportamentos, as práticas ou a identidade de gênero de uma pessoa).

Respeite os limites do paciente (linguagem não verbal). Observe algum desconforto e reformule a pergunta ou explique brevemente por que você está fazendo o questionamento, caso o paciente pareça ofendido ou relutante em responder.

Monitore e contenha suas próprias reações (linguagem não verbal).

Avise que as mesmas perguntas são feitas a todas as pessoas (procedimento protocolar), independentemente de sexo, idade, profissão ou estado civil.

Use termos neutros e inclusivos (por exemplo, "parceria" ao invés de "namorado", "namorada", "marido" ou "esposa") e faça as perguntas de forma não julgadora.

Atendendo uma pessoa transexual, pergunte como esta prefere ser chamada ou identificada. Dê suporte à identidade de gênero atual, mesmo que sua anatomia não corresponda a essa identidade.

\section{Figura 1 - Orientações gerais para abordagem da sexualidade pelos profissionais de saúde}

Atualmente, a telemedicina vem ganhando espaço na difusão de informação, promoção e prevenção, dando maior alcance aos serviços de saúde, especialmente em um país continental e com regiões de difícil acesso. Boas técnicas de comunicação no atendimento às pessoas com IST também são necessárias na telemedicina. ${ }^{14}$

Por esse motivo, é preciso incorporar sua utilização também no atendimento às pessoas com IST, respeitando os limites éticos e as recomendações atuais no que diz respeito à guarda, manuseio e transmissão de dados, assim como confidencialidade, privacidade e garantia do sigilo profissional. ${ }^{15}$

\section{Sexualidade em fases específicas da vida}

A adolescência é um período de grandes transformações biológicas, psicológicas e sociais. As alterações físicas, as interações sociais e o despertar de novos interesses refletem as mudanças rápidas e profundas que caracterizam essa etapa da vida. A maneira como os jovens expressam e vivem a sexualidade é influenciada pela qualidade das relações emocionais e afetivas vivenciadas com pessoas significativas na infância, a integração com seus pares, as transformações em decorrência do crescimento e desenvolvimento, o início da capacidade reprodutiva, as crenças, normas morais, mitos e tabus, bem como as tradições da família e da sociedade na qual estão inseridos. ${ }^{16}$

Nessa etapa, valores, atitudes, hábitos e comportamentos estão em processo de formação, transformação e solidificação, tornando os adolescentes mais vulneráveis, principalmente porque os pais ou responsáveis, a escola e a própria equipe de saúde tendem a não abordar os aspectos referentes ao exercício da sexualidade. Assim, muitas vezes os jovens iniciam a vida sexual sem as devidas orientações. ${ }^{17}$

Os serviços de saúde podem desempenhar papel fundamental, disponibilizando aos adolescentes informações que contribuam para o despertar de uma vida sexual saudável, além da prevenção das IST e da gravidez não planejada. Essas orientações devem estar pautadas no diálogo, proporcionando autonomia e atitudes responsáveis. ${ }^{18} \mathrm{~A}$ abordagem deve estar em conformidade com os princípios da confidencialidade e privacidade, indispensáveis para uma relação de confiança e respeito entre adolescentes e profissionais de saúde. ${ }^{19}$ Deve também ocorrer na perspectiva do cuidado integral, proporcionando o acesso às diferentes tecnologias associadas à prevenção combinada. ${ }^{16}$

Durante a gestação, as relações sexuais não oferecem risco à gravidez, exceto em situações obstétricas especiais (rotura de membranas, incontinência do istmo cervical, colo uterino encurtado ou trabalho de parto prematuro). Entretanto, não se pode desconsiderar a possibilidade de a gestante contrair uma IST que prejudique a evolução da gestação ou que possa ser transmitida verticalmente. Por essa razão, a equipe de saúde deve abordar rotineiramente as questões relacionadas à saúde sexual das gestantes e suas parcerias sexuais, bem como ofertar testagens para HIV, sífilis e hepatites B e C durante a assistência pré-natal. ${ }^{18}$ 
0 grupo constituído pelas pessoas idosas apresentou aumento do número de casos de HIV e de sífilis nos últimos anos, chamando atenção para a importância de se considerar o exercício da sexualidade nessa faixa etária. ${ }^{20,21}$ Há importantes aspectos que aumentam a vulnerabilidade, como menor lubrificação genital das mulheres e dificuldade de ereção masculina, dentre outros. ${ }^{20-22}$ Além disso, trata-se de uma geração que não teve iniciação sexual com práticas do sexo seguro.

\section{Avaliação de risco, vulnerabilidades e rastreamento das IST}

$\mathrm{Na}$ avaliação de risco para IST em pessoas com vida sexual ativa, recomenda-se realizar a investigação com perguntas estruturadas, visando identificar os fatores relacionados às práticas e comportamentos sexuais e uso de álcool e outras drogas. A partir dos conteúdos elucidados, será possível fazer uma avaliação adequada

\begin{tabular}{|c|c|}
\hline \multicolumn{2}{|c|}{ Sugestões de perguntas a serem feitas por profissionais de saúde às pessoas com vida sexual } \\
\hline Saúde sexual & $\begin{array}{l}\text { "Vou fazer algumas perguntas sobre sua saúde sexual. Uma vez que a saúde sexual é muito } \\
\text { importante para a saúde geral, sempre pergunto aos pacientes sobre isso. Se está tudo bem para } \\
\text { você, eu vou fazer algumas perguntas sobre questões sexuais agora. Antes de começar, você tem } \\
\text { dúvidas ou alguma preocupação em relação à sua saúde sexual que gostaria de discutir?" }\end{array}$ \\
\hline \multirow{3}{*}{ Identificação } & $\begin{array}{l}\text { "O que você se considera ser (orientação sexual)? Homossexual (gay, lésbica), heterossexual, } \\
\text { bissexual, outra, não sabe?" }\end{array}$ \\
\hline & $\begin{array}{l}\text { "Qual é a sua identidade de gênero? Homem, mulher, homem transexual, mulher transexual, } \\
\text { travesti, outra?" }\end{array}$ \\
\hline & "Com que sexo você foi designado no nascimento, conforme sua certidão de nascimento?" \\
\hline \multirow{5}{*}{ Parcerias } & "Você já teve relações sexuais?" \\
\hline & $\begin{array}{l}\text { Se sim: "Quantas parcerias sexuais você teve no último ano?" (ou em outro período de tempo, de } \\
\text { acordo com a avaliaçẫo clínica a ser realizada na consulta). }\end{array}$ \\
\hline & "Você teve relações sexuais com homens, mulheres ou ambos?" \\
\hline & $\begin{array}{l}\text { "Nos últimos três meses, você teve relações sexuais com alguém que não conhecia ou acabou de } \\
\text { conhecer?" }\end{array}$ \\
\hline & "Você já foi forçado(a) ou pressionado(a) a ter relações sexuais?" \\
\hline \multirow{3}{*}{ Práticas sexuais } & $\begin{array}{l}\text { "Nos últimos três meses, que tipos de sexo você teve? Anal? Vaginal? Oral? Receptivo (passivo), } \\
\text { insertivo (ativo), ambos (passivo e ativo)?" }\end{array}$ \\
\hline & "Você ou sua parceria usou álcool ou drogas quando você fez sexo?" \\
\hline & "Você já trocou sexo por drogas ou dinheiro?" \\
\hline \multirow{2}{*}{$\begin{array}{l}\text { História das infecções } \\
\text { sexualmente transmissiveis }\end{array}$} & $\begin{array}{l}\text { "Você já teve uma infecção sexualmente transmissível?"Se sim: "Qual? Onde foi a infecção? } \\
\text { Quando foi? Você tratou? Sua parceria se tratou?" }\end{array}$ \\
\hline & $\begin{array}{l}\text { "Você já foi testado(a) para o HIV, sífilis, hepatite B/C?" Se sim:"Há quanto tempo foi esse teste? } \\
\text { Qual foi o resultado?" }\end{array}$ \\
\hline \multirow{3}{*}{ Proteção } & "0 que você faz para se proteger das infecções sexualmente transmissíveis, incluindo o HIV?" \\
\hline & "Quando você usa essa proteção? Com quais parcerias?" \\
\hline & "Você foi vacinado contra hepatite B? Hepatite A? HPV?" \\
\hline \multirow{3}{*}{ Planejamento familiar } & "Você tem algum desejo de ter (mais) filhos(as)?" \\
\hline & $\begin{array}{l}\text { Se sim: "Quantos filhos (as) você gostaria de ter? Quando você gostaria de ter um filho? } 0 \text { que você } \\
\text { e sua parceria estão fazendo para evitar a gravidez até este momento?" }\end{array}$ \\
\hline & $\begin{array}{l}\text { Se não: "Você está fazendo alguma coisa para evitar a gravidez?" (Certifique-se de fazer as mesmas } \\
\text { perguntas também a pessoas transexuais que ainda possuem órgãos reprodutivos femininos). }\end{array}$ \\
\hline
\end{tabular}

Fonte: adaptado de Rocha et al., 2019;10 Nusbaum, Hamilton, 2002;30 Workowski, Bolan, 2015.31

Figura 2 - Perguntas da rotina de atendimento para avaliação de risco para infecções sexualmente transmissíveis 


\begin{tabular}{|c|c|c|c|c|}
\hline \multirow{2}{*}{ Subgrupos } & \multicolumn{4}{|l|}{ Periodicidade } \\
\hline & HIV $^{\mathrm{a}}$ & Sífilis $^{b}$ & $\begin{array}{l}\text { Clamídia e } \\
\text { gonocococ }\end{array}$ & Hepatites $B^{d}$ e C \\
\hline Adolescentes, jovens & \multicolumn{2}{|l|}{ Anual } & \multicolumn{2}{|c|}{$\begin{array}{l}\text { Frequência conforme outros subgrupos } \\
\text { populacionais ou práticas sexuais abaixo }\end{array}$} \\
\hline \multirow{4}{*}{ Gestantes } & \multicolumn{2}{|c|}{$\begin{array}{l}\text { Na primeira consulta do pré-natal (idealmente, } \\
\text { no } 1^{\circ} \text { trimestre da gestação); }\end{array}$} & \multirow{4}{*}{$\begin{array}{l}\text { Na primeira consulta } \\
\text { do pré-natal (para } \\
\text { gestantes } \leq 30 \text { anos) }\end{array}$} & \multirow{2}{*}{$\begin{array}{l}\text { Hepatite B: na } \\
\text { primeira consulta } \\
\text { do pré-natal } \\
\text { (idealmente, no } \\
\text { primeiro trimestre) }\end{array}$} \\
\hline & \multicolumn{2}{|c|}{ No início do $3^{\circ}$ trimestre (28a semana); } & & \\
\hline & \multicolumn{2}{|c|}{$\begin{array}{l}\text { No momento do parto, independentemente de } \\
\text { exames anteriores. }\end{array}$} & & \multirow{2}{*}{$\begin{array}{l}\text { Hepatite C: na } \\
\text { primeira consulta do } \\
\text { pré-natal }\end{array}$} \\
\hline & \multicolumn{2}{|c|}{$\begin{array}{l}\text { Em caso de aborto/natimorto, testar para sífilis, } \\
\text { independentemente de exames anteriores. }\end{array}$} & & \\
\hline $\begin{array}{l}\text { Gays e homens que fazem sexo } \\
\text { com homens }\end{array}$ & \multirow{4}{*}{\multicolumn{2}{|c|}{ Semestral }} & \multirow{4}{*}{$\begin{array}{l}\text { Ver frequência } \\
\text { conforme outros } \\
\text { subgrupos } \\
\text { populacionais ou } \\
\text { práticas sexuais }\end{array}$} & \multirow{4}{*}{ Semestral } \\
\hline Profissionais do sexo & & & & \\
\hline Travestis/transexuais & & & & \\
\hline $\begin{array}{l}\text { Pessoas que usam álcool e } \\
\text { outras drogas }\end{array}$ & & & & \\
\hline $\begin{array}{l}\text { Pessoas com diagnóstico } \\
\text { de infecção sexualmente } \\
\text { transmissível }\end{array}$ & \multicolumn{2}{|c|}{$\begin{array}{l}\text { No momento do diagnóstico e } 4 \text { a } 6 \text { semanas } \\
\text { após o diagnóstico de infecção sexualmente } \\
\text { transmissível }\end{array}$} & $\begin{array}{l}\text { No momento do } \\
\text { diagnóstico }\end{array}$ & $\begin{array}{l}\text { No momento do } \\
\text { diagnóstico }\end{array}$ \\
\hline $\begin{array}{l}\text { Pessoas com diagnóstico de } \\
\text { hepatites virais }\end{array}$ & $\begin{array}{l}\text { No momento do } \\
\text { diagnóstico }\end{array}$ & - & - & - \\
\hline $\begin{array}{l}\text { Pessoas com diagnóstico de } \\
\text { tuberculose }\end{array}$ & $\begin{array}{l}\text { No momento do } \\
\text { diagnóstico }\end{array}$ & - & - & - \\
\hline Pessoa vivendo com HIV & - & Semestral & $\begin{array}{l}\text { No momento do } \\
\text { diagnóstico }\end{array}$ & Anual \\
\hline $\begin{array}{l}\text { Pessoas com prática sexual anal } \\
\text { receptiva (passiva) sem uso de } \\
\text { preservativos }\end{array}$ & \multicolumn{4}{|l|}{ Semestral } \\
\hline Pessoas privadas de liberdade & Anual & Semestral & - & Semestral \\
\hline Violência sexual & $\begin{array}{l}\text { No atendimento } \\
\text { inicial; } 4 \text { a } 6 \text { semanas } \\
\text { após exposição } \\
\text { e } 3 \text { meses após a } \\
\text { exposição }\end{array}$ & \multicolumn{2}{|c|}{$\begin{array}{l}\text { No atendimento inicial e } 4 \text { a } 6 \text { semanas após a } \\
\text { exposição }\end{array}$} & $\begin{array}{l}\text { No atendimento } \\
\text { inicial, } 3 \text { e } 6 \text { meses } \\
\text { após a exposição }\end{array}$ \\
\hline $\begin{array}{l}\text { Pessoas em uso de profilaxia } \\
\text { pré-exposição de risco à } \\
\text { infecção pelo HIV (PrEP) }\end{array}$ & $\begin{array}{l}\text { Em cada visita ao } \\
\text { serviço }\end{array}$ & Trimestral & Semestral & Trimestral \\
\hline $\begin{array}{l}\text { Pessoas com indicação de } \\
\text { profilaxia pós-exposição à } \\
\text { infecção pelo HIV (PEP) }\end{array}$ & $\begin{array}{l}\text { No atendimento } \\
\text { inicial; } 4 \text { a } 6 \text { semanas } \\
\text { após exposição } \\
\text { e } 3 \text { meses após a } \\
\text { exposição }\end{array}$ & $\begin{array}{l}\text { No atendimento } \\
\text { inicial e } 4 \text { a } 6 \text { semanas } \\
\text { após a exposição }\end{array}$ & $\begin{array}{l}\text { No atendimento } \\
\text { inicial e } 4 \text { a } 6 \text { semanas } \\
\text { após exposição } \\
\text { (exceto nos casos de } \\
\text { acidente com material } \\
\text { biológico) }\end{array}$ & $\begin{array}{l}\text { No atendimento } \\
\text { inicial e } 6 \text { meses após } \\
\text { exposição }\end{array}$ \\
\hline
\end{tabular}

Notas: a) HIV: preferencialmente com teste rápido; b) Sífilis: preferencialmente com teste rápido; c) Clamídia e gonococo: detecção de clamídia e gonococo por biologia molecular. Pesquisa de acordo com a prática sexual: urina (uretral), amostras endocervicais, secreção genital. Para amostras extragenitais (anais e faríngeas), utilizar testes com validação para tais sítios anatômicos de coleta; d) Hepatite B: preferencialmente com teste rápido. Recomenda-se vacinar toda pessoa suscetível à hepatite B. Pessoa suscetível é aquela que não possui registro de esquema vacinal completo e que apresenta antígeno de superfície do vírus da hepatite B não reagente (ou teste rápido para hepatite B não reagente); e) Hepatite C: preferencialmente com teste rápido; f) Caso a gestante não tenha realizado rastreio no pré-natal, proceder à testagem rápida para hepatite $B$ no momento do parto. A vacina para hepatite $B$ é segura durante a gestação e as mulheres suscetíveis devem ser vacinadas.

Figura 3 - Indicação de rastreamento para infecção sexualmente transmissível de acordo com subgrupos populacionais 


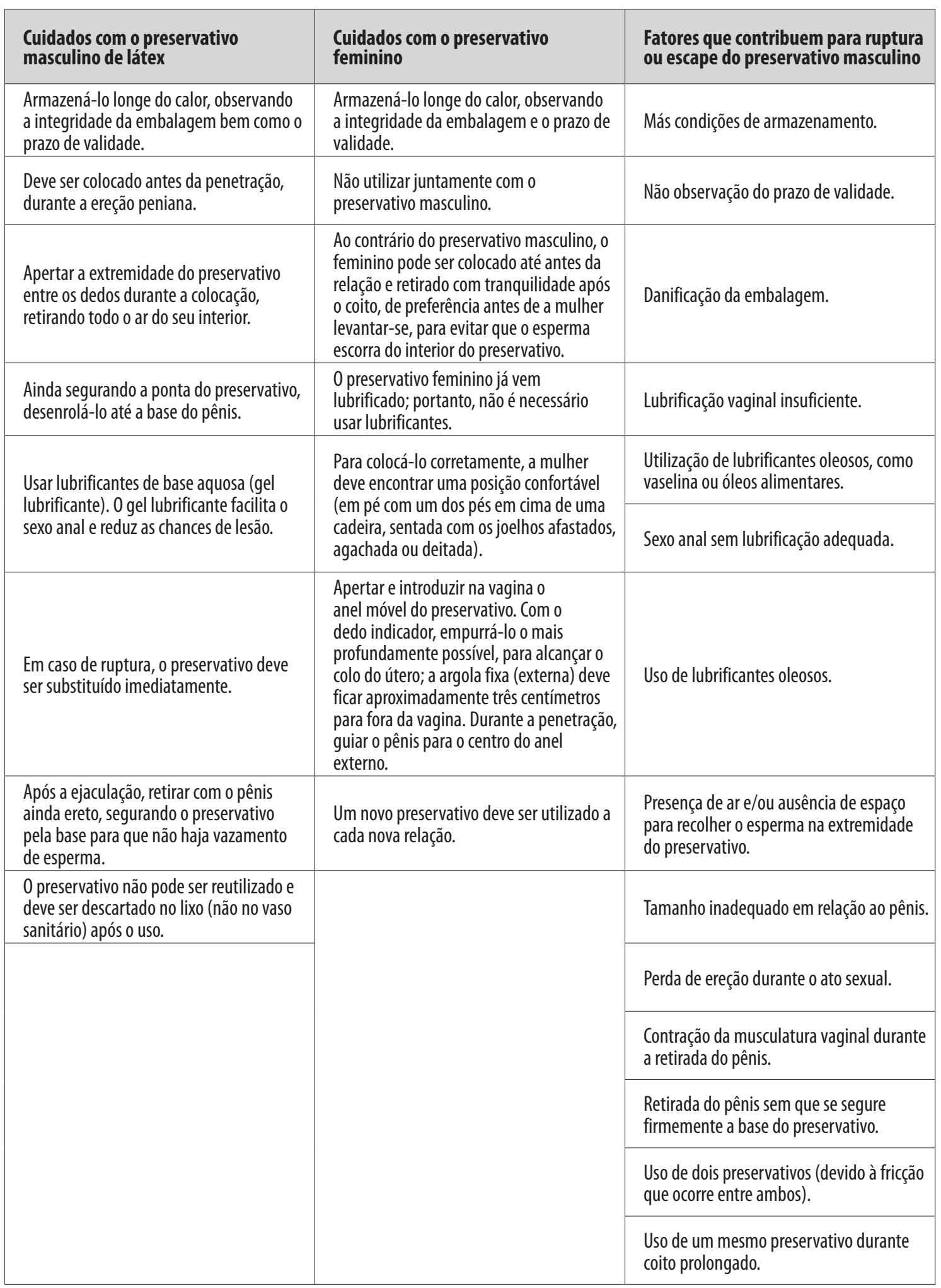

Figura 4 - Uso e conservação adequada do preservativo masculino e feminino 
para gerenciamento de risco, bem como identificar oportunidades para recomendar ações de prevenção. É importante que o profissional realize 0 atendimento de forma individualizada e em ambiente com privacidade. ${ }^{1,16,23}$ A Figura 2 apresenta perguntas para 0 atendimento voltado para avaliação de risco.

A avaliação de risco pode orientar o rastreamento das IST, e, no caso específico de pessoas diagnosticadas, é imprescindível a convocação e tratamento das parcerias sexuais, que tem como objetivo interromper a cadeia de transmissão, prevenir as complicações e impedir a reinfecção. ${ }^{1}$ No Brasil, o PCDT recomenda 0 rastreamento por meio da realização de alguns exames em pessoas assintomáticas. ${ }^{1}$ É indicado para alguns subgrupos populacionais, a fim de identificar os casos e tratá-los precocemente, buscando prevenir a disseminação das IST, bem como suas complicações. ${ }^{24}$

As diretrizes europeias orientam 0 gerenciamento das parcerias sexuais de pessoas com IST recomendando apoio emocional e identificação e notificação dos contatos, por meio de uma estratégia orientada por procedimento operacional padrão para o controle, monitoramento, tratamento e notificação dos casos. ${ }^{25}$

0 Brasil apresenta tendência de aumento de HIV e sífilis na população de 13 a 29 anos $^{20,21}$ e, por esse motivo, está indicado o rastreamento anual dessas infecções em pessoas com até 30 anos e com vida sexual ativa. Outras infecções serão triadas, a depender dos grupos populacionais e práticas sexuais que expõem as pessoas a maiores riscos. Na Figura 3, estão apresentadas as recomendações para rastreamento de pessoas de qualquer faixa etária.

É importante rastrear algumas infecções, como clamídia e gonococo, visando evitar a doença inflamatória pélvica que, mesmo assintomática, pode reduzir as chances de gravidez. ${ }^{26}$ No Brasil, recomenda-se 0 rastreamento de infecções por clamídia em gestantes abaixo de 30 anos, devido à elevada prevalência da infecção nessa faixa etária. ${ }^{27}$

Na Figura 3, constam as indicações de rastreamento para IST de acordo com subgrupos populacionais, conforme o PCDT para Atenção Integral às Pessoas com IST $2020{ }^{1}$

\section{Uso de preservativos}

0 uso de preservativo masculino ou feminino é uma estratégia de prevenção que deve ser ofertada às pessoas sexualmente ativas para a redução do risco de transmissão do HIV e de outras IST, além de evitar a gravidez. ${ }^{28}$ Apesar da baixa adesão e aceitação do preservativo feminino, acredita-se que esse insumo pode ser importante em situações nas quais existe a dificuldade de a mulher negociar o uso do preservativo masculino. ${ }^{29}$

A oferta de preservativos deve ocorrer sem restrições de quantidade e sem exigência de documentos de identificação. As orientações para conservação e uso correto dos preservativos masculino e feminino estão descritas na Figura 4 e devem fazer parte da abordagem dos profissionais de saúde em todos os atendimentos, especialmente para pessoas com maior vulnerabilidade. ${ }^{1}$

\section{Considerações finais}

É importante que os profissionais de saúde incorporem a abordagem acerca da sexualidade nos atendimentos às pessoas com vida sexual ativa, principalmente naquelas com queixas de IST. Essa abordagem deve ocorrer de forma clara, sem preconceitos ou juízos de valor, com foco na saúde sexual e reprodutiva. Dessa maneira, é possível contribuir para que as pessoas possam exercer sua sexualidade de forma plena, responsável e segura. Uma abordagem preventiva adequada pode favorecer a diminuição das IST e suas consequências.

\section{Contribuição dos autores}

Araujo MAL, Uesono J, Machado NMS, Pinto VM e Amaral E contribuíram com a concepção e delineamento do artigo, análise e interpretação dos dados e redação da primeira versão do manuscrito. Todos os autores aprovaram a versão final do trabalho e são responsáveis por todos os seus aspectos, incluindo a garantia de sua precisão e integridade.

\section{Agradecimento}

Os autores agradecem aos membros do grupo técnico de especialistas responsáveis pela elaboração do PCDT para Atenção Integral às Pessoas com IST 2020 pela contribuição substancial para a realização deste trabalho. 


\section{Referências}

1. Ministério da Saúde (BR). Secretaria de Vigilância em Saúde. Departamento de Doenças de Condições Crônicas e Infecções Sexualmente Transmissíveis. Procolo clínico e diretrizes terapeuticas para atenção integral às pessoas com infecções sexualmente transmissíveis (IST) [Internet]. Brasília: Ministério da Saúde; 2020 [citado 2020 out 29]. 248 p. Disponível em: http://www.aids.gov.br/pt-br/ pub/2015/protocolo-clinico-e-diretrizes-terapeuticas-para-atencao-integral-pessoas-com-infeccoes.

2. Ministério da Saúde (BR). Secretaria de Vigilância em Saúde. Departamento de Doenças de Condições Crônicas e Infecções Sexualmente Transmissíveis. Protocolo clínico e diretrizes terapêuticas para manejo da infecção pelo HIV em adultos [Internet]. Brasília: Ministério da Saúde; 2018 [citado 2020 out 29]. Disponível em: http://www.aids.gov.br/pt-br/ pub/2013/protocolo-clinico-e-diretrizes-terapeuticas-para-manejo-da-infeccao-pelo-hiv-em-adultos.

3. Brasil. Ministério da Saúde. Portaria MS/GM n ${ }^{\circ} 42$, de 5 de outubro de 2018. Torna pública a decisão de aprovar o Protocolo Clínico e Diretrizes Terapêuticas para Atenção Integral às Pessoas com Infecções Sexualmente Transmissíveis (IST) no âmbito do Sistema Único de Saúde - SUS [Internet]. Diário Oficial da União, Brasília (DF), 2018 out 8 [citado 2020 out 29];Seção 1:88. Disponível em: http://conitec. gov.br/images/Protocolos/PCDT_DoencaChagas.pdf.

4. Ministério da Saúde (BR). Secretaria de Atenção à Saúde. Departamento de Atenção Básica. Saúde sexual e reprodutiva [Internet]. Brasília: Ministério da Saúde; 2013 [citado 2020 out 29]. 300 p. (Cadernos de Atenção Básica, n. 26). Disponível em: https://bvsms.saude.gov.br/bvs/publicacoes/ saude_sexual_saude_reprodutiva.pdf.

5. Naciones Unidas - ONU. Informe de la Conferencia Internacional sobre la población y el desarrollo: el Cairo, 5 a 13 de septiembre de 1994 [Internet]. Nueva York: Naciones Unidas, 1995 [citado 2020 out 7]. Disponível em: https://www.unfpa.org/ sites/default/files/pub-pdf/icpd_spa.pdf.

6. Coleman E. What is sexual health? Articulating a sexual health approach to HIV prevention for men who have sex with men. AIDS Behav [Internet]. 2011 Apr [cited 2020 0ct 29];15(Suppl. 1):S18-24. Available from: https://doi.org/10.1007/s10461-011-9909-y.
7. World Health Organization - WHO. Defining sexual health: report of a technical consultation on sexual health [Internet]. Geneva: WHO; 2006 [cited 2020 0ct 29]. 35 p. Available from: https:// www.who.int/reproductivehealth/publications/ sexual_health/defining_sexual_health.pdf.

8. Creusa T, Negreiros M. Sexualidade e gênero no envelhecimento. Alceu [Internet]. 2004 jul-dez [citado 2020 out 29];5(9):77-86. Disponível em: http://revistaalceu-acervo.com. puc-rio.br/media/alceu_n9_negreiros.pdf.

9. Wei D, Xu A, Wu X. The mediating effect of trust on the relationship between doctor-patient communication and patients' risk perception during treatment. Psych J [Internet]. 2020;9(3):383-91. Available from: https://doi.org/10.1002/pchj.327.

10. Rocha AFB, Araújo MAL, Miranda AE, Leon RGP, Silva Junior GB, Vasconcelos LDPG. Management of sexual partners of pregnant women with syphilis in northeastern Brazil - a qualitative study. BMC Health Serv Res [Internet]. 2019 Jan [cited 2020 0ct 29];19(1):65. Available from: https://doi.org/10.1186/s12913-019-3910-y.

11. Carrió FB. Entrevista clínica: habilidades de comunicação para profissionais de saúde. Porto Alegre: Artmed; 2012. 346 p.

12. Toskin I, Bakunina N, Gerbase AC, Blondeel K, Stephenson R, Baggaley R, et al. A combination approach of behavioural and biomedical interventions for prevention of sexually transmitted infections. Bull World Health Organ [Internet]. 2020;98(6):431-4. Available from: http://dx.doi.org/10.2471/BLT.19.238170.

13. Kee JWY, Khoo HS, Lim I, Koh MYH. Communication skills in patient-doctor interactions: learning from patient complaints. Health Prof Educ [Internet]. 2018 Jun [cited 2020 0ct 29];4(2):97-106. Available from: https://doi.org/10.1016/j.hpe.2017.03.006.

14. Machado FSN, Carvalho MAP, Mataresi A, Mendonça ET, Cardoso LM, Yogi MS, et al. Use of telemedicine technology as a strategy to promote health care of riverside communities in the Amazon: experience with interdisciplinary work, integrating NHS guidelines. Ciênc Saúde Coletiva [Internet]. 2010;15(1):247-54. Available from: https://doi. org/10.1590/\$1413-81232010000100030. 
15. Conselho Federal de Medicina (BR). Resolução CFM $n^{0} 2.228 / 2019$. Revoga a Resolução CFM no 2.227 e reestabelece a Resolução CFM n ${ }^{0} 1.643 / 2002$, que define e disciplina a prestação de serviços através da Telemedicina [Internet]. Diário Oficial da União, Brasília (DF), 2019 mar 6 [citado 2020 out 29];Seção I:91. Disponível em: https:/www.in.gov.br/materia/-/ asset_publisher/Kujrw0TZC2Mb/content/id/65864894

16. Organização Pan-Americana da Saúde - OPAS. Organização Mundial da Saúde - OMS. Saúde e sexualidade de adolescentes. Construindo equidade no SUS. [Internet]. Brasília: Ministério da Saúde; 2017 [citado 2020 out 29]. 71 p. Disponível em: http://portalarquivos.saude.gov.br/images/PDF/2017/ maio/05/LIVRO-SAUDE-ADOLESCENTES.PDF.

17. Minstério do Planejamento, Desenvolvimento e Gestão (BR). Instituto Brasileiro de Geografia e Estatística - IBGE. Pesquisa nacional de saúde do escolar 2015 [Internet]. Rio de Janeiro: IBGE; 2016 [citado 2020 out 29]. 132 p. Disponível em: https:// biblioteca.ibge.gov.br/visualizacao/livros/liv97870.pdf.

18. Ministério da Saúde (BR). Secretaria de Vigilância em Saúde. Departamento de Doenças de Condições Crônicas e Infecções Sexualmente Transmissíveis. Protocolo clínico e diretrizes terapêuticas para prevenção da transmissão vertical de HIV, sífilis e hepatites virais [Internet]. Brasília: Ministério da Saúde; 2019 [citado 2020 out 29]. 248 p. Disponível em: http://www.aids.gov.br/pt-br/pub/2015/ protocolo-clinico-e-diretrizes-terapeuticas-para-prevencao-da-transmissao-vertical-de-hiv.

19. Sociedade Brasileira de Pediatria - SBP. Departamento Científico de Adolescência. Consulta do adolescente: abordagem clínica, orientações éticas e legais como instrumentos ao pediatra: manual de orientação [Internet]. Rio de Janeiro: SBP; 2019 [citado 2020 out 29]. Disponível em: https://www.sbp.com.br/ fileadmin/user_upload/21512c-M0_-_ConsultaAdolescente_-_abordClinica_orientEticas.pdf.

20. Ministério da Saúde (BR). Secretaria de Vigilância em Saúde. Departamento de Doenças de Condições Crônicas e Infecções Sexualmente Transmissíveis. HIV/Aids | 2019. Bol Epidemiol [Internet]. 2019 dez [citado 2020 out 29]; especial. Disponível em: http://www.aids.gov.br/pt-br/pub/2019/ boletim-epidemiologico-de-hivaids-2019

21. Ministério da Saúde (BR). Secretaria de Vigilância em Saúde. Departamento de Doenças de Condições
Crônicas e Infecções Sexualmente Transmissíveis. Sífilis | 2019. 2019 out [citado 2020 out 29];especial. Disponível em: http://www.aids.gov.br/pt-br/ pub/2019/boletim-epidemiologico-sifilis-2019.

22. Aguiar RB, Leal MCC, Marques AP0. Knowledge and attitudes about sexuality in the elderly with HIV. Ciênc Saúde Coletiva [Internet]. 2020 [cited 2020 Oct 29];25(6):2051-62. Available from: https:// doi.org.10.1590/1413-81232020256.18432018.

23. Ministério da Saúde (BR). Nota Técnica no 04, de 3 de abril de 2017. 0 direito de adolescentes serem atendidos nas UBS desacompanhados dos pais ou responsáveis e as ocasiões em que é necessária a presença de pais ou responsável. [Internet]. Brasília: Ministério da Saúde; 2017 [citado 2020 out 29]. Disponível em: https:/central3.to.gov.br/arquivo/494626/.

24. Gérvas J, Fernández MP. Uso y abuso del poder médico para definir enfermedad y factor de riesgo, en relación con la prevención cuaternaria. Gac Sanit [Internet]. 2006 Dec [cited 2020 0ct 29];20(Suppl. 3):66-71. Available from: https://doi.org/10.1157/13101092.

25. Tiplica GS, Radcliffe K, Evans C, Gomberg M, Nandwani R, Rafila A, et al. 2015 European guidelines for the management of partners of persons with sexually transmitted infections. J Eur Acad Dermatol Venereol [Internet]. 2015 [cited 2020 0ct 29];29(7):1251-7. Available from: https://doi.org/10.1111/jdv.13181.

26. Wiesenfeld HC, Hillier SL, Meyn LA, Amortegui AJ, Sweet RL. Subclinical pelvic inflammatory disease and infertility. Obstet Gynecol [Internet]. 2012 Jul [cited 2020 0ct 29];120(1):37-43. Available from: https://doi.org/10.1097/aog.0b013e31825a6bc9

27. Pinto VM, Szwarcwald CL, Baroni C, Stringari LL, Inocencio LA, Miranda AE. Chlamydia trachomatis prevalence and risk behaviors in parturient women aged 15 to 24 in Brazil. Sex Transm Dis [Internet]. 2011 Oct [cited 2020 0ct 29];38(10):957-61. Available from: https://doi.org/10.1097/olq.0b013e31822037fc.

28. Ministério da Saúde (BR). Secretaria de Vigilância em Saúde. Departamento de Vigilância, Prevenção e Controle das Infecções Sexualmente Transmissíveis, do HIV/Aids e das Hepatites Virais. Prevenção combinada do HIV: bases conceituais para profissionais, trabalhadores(as) e gestores(as) de saúde [Internet]. Brasília: Ministério da Saúde; 2017 [citado 2020 out 29]. 123 p. Disponível em: http://www.aids.gov.br/pt-br/ pub/2017/prevencao-combinada-do-hiv-bases-conceituais-para-profissionais-trabalhadoresas-e-gestores. 
29. Gomes VLO, Fonseca ADF, Jundi MG, Severo TP. Percepções de casais heterossexuais acerca do uso da camisinha feminina. Esc Anna Nery [Internet]. 2011 Jan-Mar [cited 2020 0ct 20];15(1)22-30. Available from: https://doi. org/10.1590/S1414-81452011000100004

30. Nusbaum MRH, Hamilton CD. The proactive sexual health history. Am Fam Physician [Internet]. 2002 Nov

\section{Abstract}

This article aims to present concepts and clinical practices recommended to approach people with an active sex life. These concepts are an integral part of the recommendations of the Clinical Protocol and Therapeutic Guidelines for Comprehensive Care for People with Sexually Transmitted Infections (STI), published by the Ministry of Health of Brazil in 2020. The article proposes a comprehensive approach to sexuality for bealth promotion and presents important aspects of the communication process that must develop clearly, without prejudice and judgment, with a focus on sexual and reproductive bealth. It also highlights relevant points about the exercise of sexuality at specific stages of life, recommending assessment of risks and vulnerabilities, as well as screening for STI and condom use. In this way, it is possible to contribute so that people can exercise their sexuality fully, responsibly and safely.

Keywords: Sexuality; Sexually Transmitted Infections; Disease Prevention; Diagnostic Screening Programs; Clinical Protocols; Surveillance. [cited 2020 0ct 29];66(9):1705-12. Available from: https://www.aafp.org/afp/2002/1101/p1705.html.

31. Workowski KA, Bolan GA. Sexually Transmitted Diseases Treatment Guidelines, 2015. MMWR Morb Mortal Wkly Rep [Internet]. 2015 Jun [cited 2020 Oct 29]; 64(RR3);1-137. Available from: https://www. cdc.gov/mmwr/preview/mmwrhtml/rr6403a1.htm.

\section{Resumen}

Este artículo tiene como objetivo presentar los conceptos y las prácticas clínicas recomendados para un abordaje de la persona con una vida sexual activa. Estos conceptos son parte de las recomendaciones contenidas en el Protocolo Clínico y Directrices Terapéuticas para la Atención Integral a Personas con Infecciones de Transmisión Sexual (ITS), publicado por el Ministerio de Salud de Brasil en 2020. El artículo propone un abordaje amplio de la sexualidad para la promoción de la salud. Presenta aspectos importantes del proceso de comunicación, que debe ocurrir con claridad, sin prejuicios y juicios de valor, con un enfoque en la salud sexual y reproductiva. Destaca puntos relevantes sobre el ejercicio de la sexualidad en etapas específicas de la vida, recomendando evaluación de riesgos y vulnerabilidades, así como el rastreo de ITS y el uso de condones. De esta forma, es posible contribuir para que las personas puedan ejercer su sexualidad de manera plena, responsable y segura.

Palabras clave: Sexualidad; Infecciones de Transmisión Sexual; Prevención de Enfermedades; Programas de Detección Diagnóstica; Protocolos Clínicos; Vigilancia.

Recebido em 30/07/2020

Aprovado em 23/10/2020 


\section{Errata}

No artigo "Protocolo Brasileiro para Infecções Sexualmente Transmissíveis 2020: abordagem às pessoas com vida sexual ativa", doi: 10.1590/S1679-4974202100003.esp1, Figura 3 - Indicação de rastreamento para infecção sexualmente transmissível de acordo com subgrupos populacionais, publicado no periódico Epidemiologia e Serviços de Saúde, v. 30(Esp.1):1-10, na página 5:

Onde se lia:

\begin{tabular}{|c|c|c|c|c|}
\hline \multirow{2}{*}{ Subgrupos } & $H_{I V}{ }^{a}$ & Sifilis $^{b}$ & $\begin{array}{l}\text { Clamídia e } \\
\text { gonocococ }\end{array}$ & Hepatites $B^{d}$ e C \\
\hline & \multicolumn{4}{|l|}{ Periodicidade } \\
\hline Adolescentes, jovens & \multicolumn{2}{|l|}{ Anual } & \multicolumn{2}{|c|}{$\begin{array}{l}\text { Frequência conforme outros subgrupos } \\
\text { populacionais ou práticas sexuais abaixo }\end{array}$} \\
\hline \multirow{4}{*}{ Gestantes } & \multicolumn{2}{|c|}{$\begin{array}{l}\text { Na primeira consulta do pré-natal (idealmente, } \\
\text { no } 1^{\circ} \text { trimestre da gestação); }\end{array}$} & \multirow{4}{*}{$\begin{array}{l}\text { Na primeira consulta } \\
\text { do pré-natal (para } \\
\text { gestantes } \leq 30 \text { anos) }\end{array}$} & \multirow{2}{*}{$\begin{array}{l}\text { Hepatite B: na } \\
\text { primeira consulta } \\
\text { do pré-natal } \\
\text { (idealmente, no } \\
\text { primeiro trimestre) }\end{array}$} \\
\hline & \multicolumn{2}{|c|}{ No início do $3^{\circ}$ trimestre (28a semana); } & & \\
\hline & \multicolumn{2}{|c|}{$\begin{array}{l}\text { No momento do parto, independentemente de } \\
\text { exames anteriores. }\end{array}$} & & \multirow{2}{*}{$\begin{array}{l}\text { Hepatite C: na } \\
\text { primeira consulta do } \\
\text { pré-natal }\end{array}$} \\
\hline & \multicolumn{2}{|c|}{$\begin{array}{l}\text { Em caso de aborto/natimorto, testar para sífilis, } \\
\text { independentemente de exames anteriores. }\end{array}$} & & \\
\hline $\begin{array}{l}\text { Gays e homens que fazem sexo } \\
\text { com homens }\end{array}$ & \multirow{4}{*}{\multicolumn{2}{|c|}{ Semestral }} & \multirow{4}{*}{$\begin{array}{l}\text { Ver frequência } \\
\text { conforme outros } \\
\text { subgrupos } \\
\text { populacionais ou } \\
\text { práticas sexuais }\end{array}$} & \multirow{4}{*}{ Semestral } \\
\hline Profissionais do sexo & & & & \\
\hline Travestis/transexuais & & & & \\
\hline $\begin{array}{l}\text { Pessoas que usam álcool e } \\
\text { outras drogas }\end{array}$ & & & & \\
\hline $\begin{array}{l}\text { Pessoas com diagnóstico } \\
\text { de infecção sexualmente } \\
\text { transmissível }\end{array}$ & \multicolumn{2}{|c|}{$\begin{array}{l}\text { No momento do diagnóstico e } 4 \text { a } 6 \text { semanas } \\
\text { após o diagnóstico de infecção sexualmente } \\
\text { transmissível }\end{array}$} & $\begin{array}{l}\text { No momento do } \\
\text { diagnóstico }\end{array}$ & $\begin{array}{l}\text { No momento do } \\
\text { diagnóstico }\end{array}$ \\
\hline $\begin{array}{l}\text { Pessoas com diagnóstico de } \\
\text { hepatites virais }\end{array}$ & $\begin{array}{l}\text { No momento do } \\
\text { diagnóstico }\end{array}$ & - & - & - \\
\hline $\begin{array}{l}\text { Pessoas com diagnóstico de } \\
\text { tuberculose }\end{array}$ & $\begin{array}{l}\text { No momento do } \\
\text { diagnóstico }\end{array}$ & - & - & - \\
\hline Pessoa vivendo com HIV & \multicolumn{2}{|l|}{ Semestral } & $\begin{array}{l}\text { No momento do } \\
\text { diagnóstico }\end{array}$ & Anual \\
\hline $\begin{array}{l}\text { Pessoas com prática sexual anal } \\
\text { receptiva (passiva) sem uso de } \\
\text { preservativos }\end{array}$ & \multicolumn{4}{|l|}{ Semestral } \\
\hline Pessoas privadas de liberdade & Anual & Semestral & - & Semestral \\
\hline Violência sexual & $\begin{array}{l}\text { No atendimento } \\
\text { inicial; } 4 \text { a } 6 \text { semanas } \\
\text { após exposição } \\
\text { e } 3 \text { meses após a } \\
\text { exposição }\end{array}$ & \multicolumn{2}{|c|}{$\begin{array}{l}\text { No atendimento inicial e } 4 \text { a } 6 \text { semanas após a } \\
\text { exposição }\end{array}$} & $\begin{array}{l}\text { No atendimento } \\
\text { inicial, } 3 \text { e } 6 \text { meses } \\
\text { após a exposição }\end{array}$ \\
\hline
\end{tabular}


Continuação

\begin{tabular}{|c|c|c|c|c|}
\hline \multirow{2}{*}{ Subgrupos } & HIV & Sífilis ${ }^{b}$ & $\begin{array}{l}\text { Clamídia e } \\
\text { gonococo }\end{array}$ & Hepatites Bd e C \\
\hline & \multicolumn{4}{|l|}{ Periodicidade } \\
\hline $\begin{array}{l}\text { Pessoas em uso de profilaxia } \\
\text { pré-exposição de risco à } \\
\text { infecção pelo HIV (PrEP) }\end{array}$ & $\begin{array}{l}\text { Em cada visita ao } \\
\text { serviço }\end{array}$ & Trimestral & Semestral & Trimestral \\
\hline $\begin{array}{l}\text { Pessoas com indicação de } \\
\text { profilaxia pós-exposição à } \\
\text { infecção pelo HIV (PEP) }\end{array}$ & $\begin{array}{l}\text { No atendimento } \\
\text { inicial; } 4 \text { a } 6 \text { semanas } \\
\text { após exposição } \\
\text { e } 3 \text { meses após a } \\
\text { exposição }\end{array}$ & $\begin{array}{l}\text { No atendimento } \\
\text { inicial e } 4 \text { a } 6 \text { semanas } \\
\text { após a exposição }\end{array}$ & $\begin{array}{l}\text { No atendimento } \\
\text { inicial e } 4 \text { a } 6 \text { semanas } \\
\text { após exposição } \\
\text { (exceto nos casos de } \\
\text { acidente com material } \\
\text { biológico) }\end{array}$ & $\begin{array}{l}\text { No atendimento } \\
\text { inicial e } 6 \text { meses após } \\
\text { exposição }\end{array}$ \\
\hline
\end{tabular}

Leia-se:

\begin{tabular}{|c|c|c|c|c|}
\hline \multirow[b]{2}{*}{ Subgrupos } & \multicolumn{4}{|c|}{ Periodicidade } \\
\hline & HIV & Sífilis ${ }^{b}$ & $\begin{array}{l}\text { Clamídia e } \\
\text { gonocococ }\end{array}$ & Hepatites Bd e C \\
\hline Adolescentes, jovens & \multicolumn{2}{|l|}{ Anual } & \multicolumn{2}{|c|}{$\begin{array}{l}\text { Frequência conforme outros subgrupos } \\
\text { populacionais ou práticas sexuais abaixo }\end{array}$} \\
\hline \multirow{4}{*}{ Gestantes } & \multicolumn{2}{|c|}{$\begin{array}{l}\text { Na primeira consulta do pré-natal (idealmente, } \\
\text { no } 1^{\circ} \text { trimestre da gestação); }\end{array}$} & \multirow{4}{*}{$\begin{array}{l}\text { Na primeira consulta } \\
\text { do pré-natal (para } \\
\text { gestantes } \leq 30 \text { anos) }\end{array}$} & \multirow{2}{*}{$\begin{array}{l}\text { Hepatite B: na } \\
\text { primeira consulta } \\
\text { do pré-natal } \\
\text { (idealmente, no }^{\text {primeiro trimestre) }}{ }^{f}\end{array}$} \\
\hline & \multicolumn{2}{|c|}{ No início do $3^{0}$ trimestre (28a semana); } & & \\
\hline & \multicolumn{2}{|c|}{$\begin{array}{l}\text { No momento do parto, independentemente de } \\
\text { exames anteriores. }\end{array}$} & & \multirow{2}{*}{$\begin{array}{l}\text { Hepatite C: na } \\
\text { primeira consulta do } \\
\text { pré-natal }\end{array}$} \\
\hline & \multicolumn{2}{|c|}{$\begin{array}{l}\text { Em caso de aborto/natimorto, testar para sífilis, } \\
\text { independentemente de exames anteriores. }\end{array}$} & & \\
\hline $\begin{array}{l}\text { Gays e homens que fazem sexo } \\
\text { com homens }\end{array}$ & \multirow{4}{*}{\multicolumn{2}{|c|}{ Semestral }} & \multirow{4}{*}{$\begin{array}{l}\text { Ver frequência } \\
\text { conforme outros } \\
\text { subgrupos } \\
\text { populacionais ou } \\
\text { práticas sexuais }\end{array}$} & \multirow{4}{*}{ Semestral } \\
\hline Profissionais do sexo & & & & \\
\hline Travestis/transexuais & & & & \\
\hline $\begin{array}{l}\text { Pessoas que usam álcool e } \\
\text { outras drogas }\end{array}$ & & & & \\
\hline $\begin{array}{l}\text { Pessoas com diagnóstico } \\
\text { de infecção sexualmente } \\
\text { transmissível }\end{array}$ & \multicolumn{2}{|c|}{$\begin{array}{l}\text { No momento do diagnóstico e } 4 \text { a } 6 \text { semanas } \\
\text { após o diagnóstico de infecção sexualmente } \\
\text { transmissível }\end{array}$} & $\begin{array}{l}\text { No momento do } \\
\text { diagnóstico }\end{array}$ & $\begin{array}{l}\text { No momento do } \\
\text { diagnóstico }\end{array}$ \\
\hline $\begin{array}{l}\text { Pessoas com diagnóstico de } \\
\text { hepatites virais }\end{array}$ & $\begin{array}{l}\text { No momento do } \\
\text { diagnóstico }\end{array}$ & - & - & - \\
\hline $\begin{array}{l}\text { Pessoas com diagnóstico de } \\
\text { tuberculose }\end{array}$ & $\begin{array}{l}\text { No momento do } \\
\text { diagnóstico }\end{array}$ & - & - & - \\
\hline Pessoa vivendo com HIV & - & Semestral & $\begin{array}{l}\text { No momento do } \\
\text { diagnóstico }\end{array}$ & Anual \\
\hline $\begin{array}{l}\text { Pessoas com prática sexual anal } \\
\text { receptiva (passiva) sem uso de } \\
\text { preservativos }\end{array}$ & \multicolumn{4}{|l|}{ Semestral } \\
\hline Pessoas privadas de liberdade & Anual & Semestral & - & Semestral \\
\hline
\end{tabular}

Continua 
Continuação

\begin{tabular}{|c|c|c|c|c|}
\hline \multirow{2}{*}{ Subgrupos } & \multicolumn{4}{|c|}{ Periodicidade } \\
\hline & HIV ${ }^{a}$ & Sífilis $^{b}$ & $\begin{array}{l}\text { Clamídia e } \\
\text { gonocococ }\end{array}$ & Hepatites $B^{d}$ e C \\
\hline Violência sexual & $\begin{array}{l}\text { No atendimento } \\
\text { inicial; } 4 \text { a } 6 \text { semanas } \\
\text { após exposição } \\
\text { e } 3 \text { meses após a } \\
\text { exposição }\end{array}$ & \multicolumn{2}{|c|}{$\begin{array}{l}\text { No atendimento inicial e } 4 \text { a } 6 \text { semanas após a } \\
\text { exposição }\end{array}$} & $\begin{array}{l}\text { No atendimento } \\
\text { inicial, } 3 \text { e } 6 \text { meses } \\
\text { após a exposição }\end{array}$ \\
\hline $\begin{array}{l}\text { Pessoas em uso de profilaxia } \\
\text { pré-exposição de risco à } \\
\text { infecção pelo HIV (PrEP) }\end{array}$ & $\begin{array}{l}\text { Em cada visita ao } \\
\text { serviço }\end{array}$ & Trimestral & Semestral & Trimestral \\
\hline $\begin{array}{l}\text { Pessoas com indicação de } \\
\text { profilaxia pós-exposição à } \\
\text { infecção pelo HIV (PEP) }\end{array}$ & $\begin{array}{l}\text { No atendimento } \\
\text { inicial; } 4 \text { a } 6 \text { semanas } \\
\text { após exposição } \\
\text { e } 3 \text { meses após a } \\
\text { exposição }\end{array}$ & $\begin{array}{l}\text { No atendimento } \\
\text { inicial e } 4 \text { a } 6 \text { semanas } \\
\text { após a exposição }\end{array}$ & $\begin{array}{l}\text { No atendimento } \\
\text { inicial e } 4 \text { a } 6 \text { semanas } \\
\text { após exposição } \\
\text { (exceto nos casos de } \\
\text { acidente com material } \\
\text { biológico) }\end{array}$ & $\begin{array}{l}\text { No atendimento } \\
\text { inicial e } 6 \text { meses após } \\
\text { exposição }\end{array}$ \\
\hline
\end{tabular}

